\title{
SIEMENS ADVANCED QUANTRA FTICR MASS SPECTROMETER FOR ULTRA HIGH RESOLUTION AT LOW MASS
}

\author{
W.A. Spencer and L. L. Tovo
}

July 2008

Analytical Development Section

Savannah River National Laboratory

Aiken, SC 29808

Prepared for the U.S. Department of Energy Under Contract Number

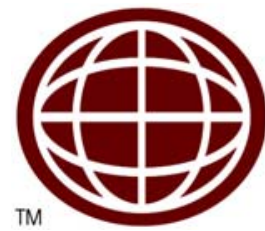




\title{
DISCLAIMER
}

This report was prepared by Westinghouse Savannah River Company (WSRC) for the United States Department of Energy under Contract No. DE-AC09-96SR18500 and is an account of work performed under that contract. Neither the United States Department of Energy, nor WSRC, nor any of their employees makes any warranty, expressed or implied, or assumes any legal liability or responsibility for the accuracy, completeness, or usefulness, of any information, apparatus, or product or process disclosed herein or represents that its use will not infringe privately owned rights. Reference herein to any specific commercial product, process, or service by trademark, name, manufacturer or otherwise does not necessarily constitute or imply endorsement, recommendation, or favoring of same by WSRC or by the United States Government or any agency thereof. The views and opinions of the authors expressed herein do not necessarily state or reflect those of the United States Government or any agency thereof.

\author{
Printed in the United States of America \\ Prepared For \\ U.S. Department of Energy
}


Key Words:

Mass spectrometer,

HD gas analysis

Retention: Permanent

\title{
SIEMENS ADVANCED QUANTRA FTICR MASS SPECTROMETER FOR ULTRA HIGH RESOLUTION AT LOW MASS
}

\author{
W.A. Spencer and L. L. Tovo
}

July 2008 


\section{REVIEWS AND APPROVALS}

\section{$\operatorname{AUTHOR(S):~}$}

W.A. Spencer, Analytical Development

Date

L. L. Tovo, Analytical Development

Date

\section{TECHNICAL REVIEWER:}

J.L. Venzie, Analytical Development

Date

\section{APPROVERS}

L. M. Chandler, Manager, Analytical Development

Date

M. J. Barnes, Manager, Spectroscopy and Separations

Date

P. F. Cloessner, Manager, Defense Programs Technology

Date 


\section{EXECUTIVE SUMMARY}

\section{- Purpose \& General Approach}

The Siemens Advanced Quantra Fourier Transform Ion Cyclotron Resonance (FTICR) mass spectrometer was evaluated as an alternative instrument to large double focusing mass spectrometers for gas analysis. High resolution mass spectrometers capable of resolving the common mass isomers of the hydrogen isotopes are used to provide data for accurate loading of reservoirs and to monitor separation of tritium, deuterium, and helium. Conventional double focusing magnetic sector instruments have a resolution that is limited to about 5000 . The Siemens FTICR instrument achieves resolution beyond 400,000 and could possibly resolve the tritium ion from the helium-3 ion, which differ by the weight of an electron, $0.00549 \mathrm{amu}$. Working with Y-12 and LANL, SRNL requested Siemens to modify their commercial Quantra system for low mass analysis. To achieve the required performance, Siemens had to increase the available waveform operating frequency from $5 \mathrm{MHz}$ to $40 \mathrm{MHz}$ and completely redesign the control electronics and software. However, they were able to use the previous ion trap, magnet, passive pump, and piezo-electric pulsed inlet valve design. NNSA invested \$1M in this project and acquired four systems, two for Y-12 and one each for SRNL and LANL. Siemens claimed a $\$ 10 \mathrm{M}$ investment in the Quantra systems.

\section{- Conclusions}

The new Siemens Advanced Quantra demonstrated phenomenal resolution in the low mass range. Resolution greater than 400,000 was achieved for mass 2 . The new spectrometer had a useful working mass range to 500 Daltons. However, experiments found that a continuous single scan from low mass to high was not possible. Two useful working ranges were established covering masses 1 to 6 and masses 12 to 500 for our studies. A compromise performance condition enabled masses 1 to 45 to be surveyed. The instrument was found to have a dynamic range of about three orders of magnitude and quantitative analysis is expected to be limited to around 5 percent without using complex fitting algorithms.

Analysis of low concentration ions, at the ppm level, required a separate analysis using ion ejection techniques. Chemical ionization due to the formation of the $\mathrm{MH}^{+}$ion or $\mathrm{MD}^{+}$increased the complexity of the spectra compared to magnetic sector mass spectra and formation of the protonated or deuterated complex was a dynamic function of the trap ion concentration. This made quantitative measurement more of a challenge.

However, the resolution of the instrument was far superior to any other mass spectrometry technique that has been applied to the analysis of the hydrogen isotopes. The piezo-electric picoliter injection device offers a new way of submitting small quantities of atmospheric pressure sample gas for analysis. The new software had many improvements over the previous version but significant flaws in the beta codes remain that make the prototype units less than ideal. The instrument is a promising new technology that experience will likely improve. Unfortunately, Siemens has concluded that the technology will not be a commercial success and has decided to stop producing this product. 


\section{- Recommendations}

Siemens has notified NNSA that they would not support the Quantra unless they found a market that would support significant annual sales. Because Siemens does not intend to support the instrument as a commercial offering, SRNL recommends limiting development work with the instrument.

The Quantra will be most useful as a complement to, not a replacement for, the existing mass spectrometers. Because of the instrument's superior resolution, it would be especially beneficial for measuring analytes that are difficult to resolve with the currently used mass spectrometers. For example, it could be used to monitor $\mathrm{CO}$ and $\mathrm{N}_{2}$ concentrations directly, which is a common support request and a very difficult conventional mass spectrometry measurement. The instrument can be used to measure mass 16-20 isomers such as oxygen, ammonia, water, or methane. For measurement of the hydrogen isotopes as well as other analytes in a hydrogen isotope matrix, method development and parameter optimization will be required to understand and minimize the chemical ionization that occurs in the ion trap. Testing with tritium will be needed to evaluate and understand its interactions in the ion trap. Finally, the Siemens Quantra may be used for training and demonstration of high resolution residual gas mass measurements with ion traps.

Development of quantitative algorithms and more robust crash resistant operating codes would make the Quantra system suitable for process support applications. Siemens will likely have at least one revision of their software to evaluate. An alternative for Quantra instrument control and data analysis would be to apply the Predator System developed at the National Magnetic Laboratory at Florida State University.

SRNL will monitor external developments and literature with Siemens and other manufacturers of FTICR systems. While this report was being reviewed, Ivey and Hemminger published a paper characterizing the issues associated with the detection of hydrogen by FTICR which are in agreement with the SRNL observations discussed in this report. The authors observed and discussed two mechanisms that affect the hydrogen signal: 1) z-ejection and 2) proton and charge-transfer reactions in the trap. They also demonstrated that parameters could be optimized to minimize these effects for quantitative measurement of hydrogen. 
TABLE OF CONTENTS

EXECUTIVE SUMMARY iii

LIST OF FIGURES vi

LIST OF TABLES vii

LIST OF ACRONYMS

1.0 INTRODUCTION AND BACKGROUND

2.0 EXPERIMENTAL

3.0 RESULTS

4.0 CONCLUSIONS

vii

5.0 RECOMMENDATIONS/PATH FORWARD

6.0 REFERENCES

7.0 ACKNOWLEDGEMENTS 


\section{LIST OF FIGURES}

Figure 1 Siemens Advanced Quantra uses a capillary inlet system with a piezo electric valve leading to a small Fourier Transform Ion Cyclotron Resonance spectrometer that is tuned for low mass trapping and detection.

Figure 2 The new client computer interface shows spectra and time trend data on a complex viewing screen. 6

Figure 3 Resonance frequency as a function of mass.................................................................... 7

Figure 4 Resolution of ${ }^{4} \mathrm{He}$ from $\mathrm{D}_{2}$ was far superior compared to other mass spectrometers in the laboratory............................................................................... 9

Figure 5 Hydrogen response was less than deuterium in the equilibrium blend of equal portions of the gases. This might be a result of ionization but more likely the hydrogen was not being confined in the trap as well as the deuterium.

Figure 6 The molecular ion tended to protonate $\left[\mathrm{MH}^{+}\right]$or deuterate $\left[\mathrm{MD}^{+}\right]$ when the trap was overloaded or a reactive species was present. Note the presence of ions at mass 5 and 6 in the mix of hydrogen and deuterium. Background noise level in this scan could easily be reduced using multiple co-added scans.

Figure 7 Minor isotopes of krypton and their relative abundance are shown; $\mathrm{m} 78 / 0.35 \%$, m80/2.28\%, m82 11.6\%, m83/ 11.5\%, m84/ 57.0\% [off scale], m86/17.4\%. This image had 128 co-adds to decrease noise. Masses calibration is slightly shifted.

Figure 8 Spectrum shows a mixture of $\mathrm{H}_{2}, \mathrm{HD},{ }^{4} \mathrm{He}$ and $\mathrm{D}_{2}$. 13

Figure 9 A close look at trend data taken at high resolution shows that the spectrometer would occasionally lose its mass lock and create a single point spike in the data set. 


\section{LIST OF TABLES}

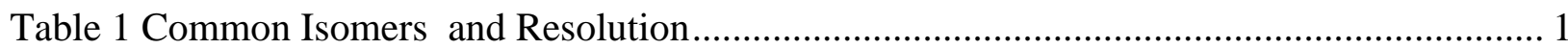

Table 2 Optimum Conditions for Mass Ranges..................................................................... 8

\section{LIST OF ACRONYMS}

$\begin{array}{ll}\text { FTICR } & \text { Fourier Transform Ion Cyclotron Resonance } \\ \text { HDT } & \text { Hydrogen Deuterium Tritium } \\ \text { HR-MS } & \text { High Resolution Mass Spectrometry } \\ \text { HWHM } & \text { Half Width at Half Maximum } \\ \text { M }^{+} & \text {Molecular Ion } \\ \text { MH }^{+} & \text {Protonated Molecular Ion } \\ \text { MD }^{+} & \text {Deuterated Molecular Ion } \\ \text { NNSA } & \text { National Nuclear Security Administration } \\ \text { ppb } & \text { part per billion } \\ \text { ppm } & \text { part per million } \\ \text { SNR } & \text { signal-to-noise ratio }\end{array}$




\subsection{INTRODUCTION AND BACKGROUND}

High resolution, low mass spectrometry is used by the Department of Energy to support measurement of hydrogen isotopes and for monitoring the separation of hydrogen, deuterium, tritium, and helium. Table 1 lists some of the common mass isomers of interest. Most of these ions can be measured using an instrument supporting a 2,500 resolution. In NNSA facilities, the hydrogen isotope measurements are generally made using either the VG-Isotopes, GAZAB double focusing mass spectrometer or the Varian/Finnigan MAT 250/271 series single focusing gas mass spectrometer. Both instruments have excellent performance specifications. These instruments were developed for DOE in the early 1980s as part of a DOE complex initiative. The primary goal of the DOE development program was to have an instrument with a resolution greater than 2000 at mass 4 and abundance sensitivity greater than 100,000. Phil Chastagner of SRNL, who was a member of the technical development team, documented the development effort and the evaluation of these spectrometers [ref 1]. In doing so, he set performance criteria for future evaluation and testing of mass spectrometers for DOE hydrogen isotope tasks. Currently, the VG-Isotopes, GAZAB and the Varian/Finnigan MAT 250/271 instruments are no longer being produced and have limited manufacturer support.

Table 1 Common Isomers and Resolution

\begin{tabular}{|c|c|c|c|}
\hline \multicolumn{2}{|c|}{ Important Ions in the Mass 2-6 Range } & \multirow[b]{2}{*}{ Resolution $(\mathbf{M} / \Delta \mathbf{M})$} & \\
\hline Ion (positive) & $\mathbf{M} / \mathbf{e}$ & & \\
\hline D & 2.01400 & 1221 & $\mathbf{D} \leftrightarrow \mathbf{H}_{2}$ \\
\hline $\mathrm{H}_{2}$ & 2.01565 & & \\
\hline${ }^{3} \mathrm{He}$ & 3.01603 & 150802 & ${ }^{3} \mathrm{He} \leftrightarrow \mathrm{T}$ \\
\hline $\mathbf{T}$ & 3.01605 & 522 & $\mathbf{T} \leftrightarrow \mathbf{H D}$ \\
\hline HD & 3.02183 & 1831 & $\mathbf{H D} \leftrightarrow \mathrm{H}_{3}$ \\
\hline $\mathbf{H}_{3}$ & 3.02348 & & \\
\hline${ }^{4} \mathrm{He}$ & 4.00260 & 188 & ${ }^{4} \mathrm{He} \leftrightarrow \mathrm{HT}$ \\
\hline HT & 4.02388 & 975 & $\mathbf{H T} \leftrightarrow \mathbf{D}_{2}$ \\
\hline $\mathbf{D}_{2}$ & 4.02800 & 2299 & $\mathbf{H T} \leftrightarrow \mathrm{H}_{2} \mathrm{D}$ \\
\hline$\overline{\mathrm{H}_{2} \mathrm{D}}$ & 4.02975 & & \\
\hline$\overline{\text { DT }}$ & 5.03005 & 731 & $\mathbf{D T} \leftrightarrow \mathbf{D}_{2} \mathbf{H}$ \\
\hline $\mathrm{D}_{2} \mathrm{H}$ or HDD & 5.03693 & & \\
\hline $\mathbf{T}_{2}$ & 6.03200 & 603 & $\mathbf{T}_{2} \leftrightarrow \mathbf{D}_{3}$ \\
\hline $\mathbf{D}_{3}$ & 6.04200 & & \\
\hline *above taken & gner, ASMS presenta & ion (1984) & \\
\hline
\end{tabular}




\begin{tabular}{|c|c|c|c|}
\hline \multicolumn{2}{|c|}{ Common Isomer Ions in the Mass 16- 44 Range } & \multirow[b]{2}{*}{ Resolution $(\mathbf{M} / \Delta \mathbf{M})$} & \\
\hline Ion (positive) & $\mathbf{M} / \mathbf{e}$ & & \\
\hline $\mathbf{N}$ & 14.00307 & & \\
\hline $\mathrm{CH}_{2}$ & 14.02156 & & \\
\hline $\mathbf{O}$ & 15.99490 & & \\
\hline $\mathbf{N H}_{2}$ & 16.01872 & & \\
\hline $\mathrm{CH}_{4}$ & 16.03130 & & \\
\hline $\mathbf{O H}$ & 17.00270 & 714 & $\mathbf{O H} \leftrightarrow \mathbf{N H}_{3}$ \\
\hline $\mathrm{NH}_{3}$ & 17.02650 & & \\
\hline $\mathbf{H}_{2} \mathbf{O}$ & 18.01060 & 758 & $\mathrm{H}_{2} \mathbf{0} \leftrightarrow \mathrm{NH}_{4}$ \\
\hline $\mathbf{N H}_{4}$ & 18.03437 & & \\
\hline HD0 & 19.01680 & & \\
\hline $\mathbf{D}_{\mathbf{2}} \mathbf{0}$ & 20.02300 & & \\
\hline $\mathrm{CO}$ & 27.99490 & 2489 & $\mathrm{CO} \leftrightarrow \mathrm{N}_{2}$ \\
\hline $\mathbf{N}_{2}$ & 28.00615 & 1118 & $\mathbf{N}_{2} \leftrightarrow \mathrm{C}_{2} \mathrm{H}_{4}$ \\
\hline $\mathrm{C}_{2} \mathrm{H}_{4}$ & 28.03120 & & \\
\hline $\mathbf{O}_{2}$ & 31.98980 & & \\
\hline$\overline{\mathbf{A r}}$ & 39.96240 & & \\
\hline $\mathrm{CO}_{2}$ & 43.98980 & 3907 & $\mathrm{CO}_{2} \leftrightarrow \mathrm{N}_{2} \mathrm{O}$ \\
\hline $\mathbf{N}_{2} \mathbf{O}$ & 44.00106 & & \\
\hline $\mathrm{C}_{2} \mathrm{H}_{4} \mathrm{O}$ & 44.02620 & & \\
\hline $\mathrm{C}_{3} \mathrm{H}_{8}$ & 44.06250 & & \\
\hline
\end{tabular}

A couple of papers have added to the discussion of hydrogen isotope measurement. Brand and Coplen reported an inter-laboratory study that compared 40 hydrogen isotopic mass spectrometer instruments. Most of the instruments in their study were either the VG or MAT instrument. [ref 2]. Sessions, Burgoyne, and Hayes found that hydrogen isotopic measurements often failed to correct adequately for the amount of $\mathrm{H}_{3}{ }^{+}$contribution [ref 3]. Demange, Grivet, Pialot, and Chambaudet published a method that indirectly determined tritium based on trapping of the hydrogen isotopes and then measuring the residual helium-3 and helium-4 ions using a sensitive helium leak detector. Their technique was interesting because the detection limits for helium were in the low ppb range [ref 4].

Because the existing instruments have limited vendor support and need an electronic and software upgrade to accommodate modern data handling, security, and new data reduction algorithms, SRNL initiated a two-fold upgrade approach. One approach was to seek alternate mass spectrometers that would take advantage of recent mass spectrometry developments and the other was to retrofit the existing mass spectrometers with newer electronics. The latter approach was also chosen at Y-12 where one of their older spectrometers was retrofitted with new electronics. Joe Cordaro and John McIntosh recently reported on the progress of the electronic upgrade of a Varian/Finnigan MAT instrument [ref 5].

As possible alternative instruments, SRNL identified two new commercial mass spectrometers that might be able to provide support for the hydrogen isotope analyses and help relieve the load on the production mass spectrometers at a lower cost. These instruments were the JEOL GCMATE and the Siemens Advanced Quantra. The GCMATE is a compact double focusing mass spectrometer and 
basically a modern variation of the existing magnetic sector instruments. Both the GCMATE and a first generation Quantra system was purchased and evaluated by SRNL with encouraging results [ref 6]. Figure 1 shows a photograph of the Quantra system.

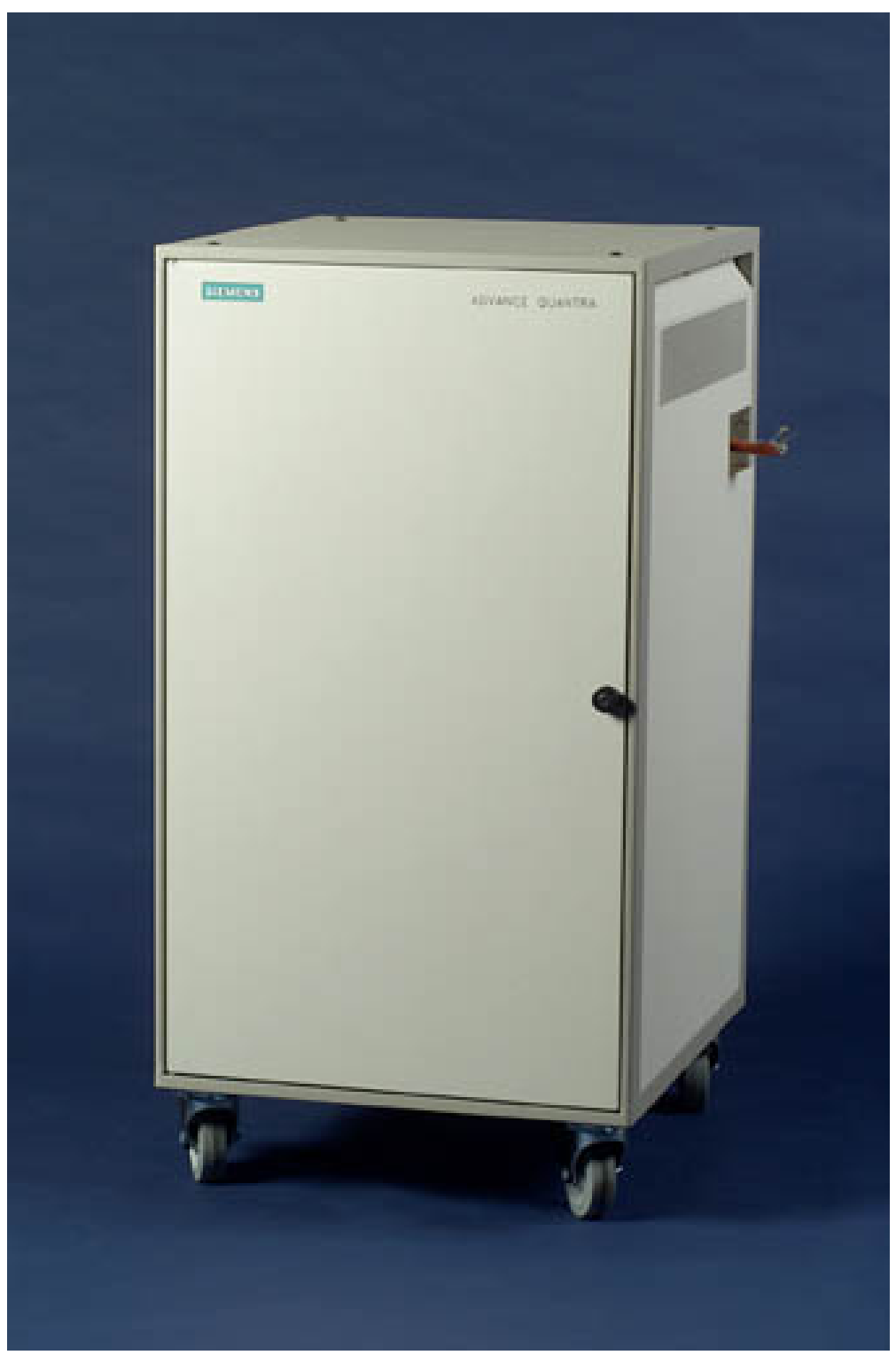

Figure 1 Siemens Advanced Quantra uses a capillary inlet system with a piezo electric valve leading to a small Fourier Transform Ion Cyclotron Resonance spectrometer that is tuned for low mass trapping and detection.

The Siemens Quantra system was an entirely new type of spectrometer for high resolution gas analyses built on the FTICR concept. Excellent tutorials on the FTICR method are available. [ref 7,8,9]. Unlike most FTICR instruments which use massive magnets for confining large molecules, the Siemens Quantra used a smaller 1 Tesla field strength magnet which was more suitable for confining the smaller volatile lower mass ions and allowed reasonable voltages for low mass ion control. The Quantra was originally designed to support the petroleum industry with high resolution separation of mass 6 to 500 . The system was designed by the Applied Automation Group which was originally part of the R\&D 
laboratory at Phillips Petroleum. Applied Automation was subsequently acquired by Siemens. The Quantra was compatible with the Applied Automation process gas chromatographic systems and could be used as a high resolution detector for the process GC. The system was made rugged, portable, and the price was relatively low compared to the magnetic sector instruments.

The Quantra includes several unique features. Instead of a molecular leak used in the inlet systems for magnetic sector mass spectrometers, the Quantra uses a piezo-electric driven valve that injects picoliter amounts of gas sampled directly from sixteenth inch capillary tubing. A voltage waveform is applied to the valve to adjust the amount of gas injected as well as the duration of the injection. Injection size is limited by the ion trap capacity.

A key feature of the Quantra, as described by Q. Grindstaff of the Y-12 National Security Complex, is that the Quantra uses an external electron beam to perform ionization in the trap [ref 11, 12]. The electron beam passes through a hole in the magnet and is thus confined as well as isolated. This gives the Quantra an advantage in that the spectrometer does not observe ions resulting from reactions of gases on the ionization filament. That is, there is very little residual water or hydrogen ion background in the Siemens Quantra system. Observation of filament generated water is a common problem observed in many mass spectrometer sources and in most residual gas analyzers. The Quantra is able to isolate the water and hydrogen formed at the filament from the ionization chamber and ion trap.

Because gas is not continuously injected into the Quantra, Siemens was able to use a very low maintenance passive pumping system. The entire system is pre-pumped to the $10^{-10}$ Torr range and then held at that level using a small titanium getter.

The original Quantra system was limited to measurement of masses above 12 AMU, because the electronics used to drive the system were limited to a maximum $5 \mathrm{MHz}$ data collection and pulse frequency. SRNL, LANL, and Y-12 worked with Dean Davis, a senior analytical chemist at Siemens Energy and Automation, Inc., to create a new instrument specification which would modify the Quantra to include low mass (1-12 amu) analyses. Davis had already shown that the $5 \mathrm{MHz}$ commercial system could trap hydrogen and helium using second harmonics but only over a very small mass range. Davis believed that an instrument could be built that used higher data rates to directly capture the hydrogen isotope cyclotron frequencies. NNSA contracted with Siemens to build four systems with a $40 \mathrm{MHz}$ converter.

The following describes the instrument specifications for the low mass FTICR system, the instrument configuration that Siemens delivered, and some of the initial observations made at SRNL with this new instrument. In addition to the evaluations being performed at SRNL, the instrument is also currently under field evaluation at LANL and at Y-12. Robert Ellefson, Mound Technical Solutions, served as an independent consultant to evaluate the Siemens development effort. 


\subsection{EXPERIMENTAL}

\section{NNSA Low Mass FTICR Request}

To acquire a new spectrometer, the collaborating sites prepared a procurement specification based on features of the original commercial Quantra, the specifications of the current VG and Finnigan mass spectrometers, as well as additional features needed to support analysis of hydrogen isotopes [ref 10].

\section{Siemens FTICR Mass Spectrometer -Configuration}

\section{Basic Configuration}

The Quantra configuration has been described in a previous report [ref 6]. Except for electronics and software, the new generation Quantra uses most of the same hardware and looks identical to the previous generation. That is the ion trap, getter pumping system, inlet system and instrument chassis/case are the same. The Quantra still has a controller computer that is located within the mass spectrometer and runs the LINUX operating system. The controller is linked to a client computer via Ethernet cabling. The client computer runs a user interface under the Microsoft Windows environment as shown in Figure 2. Both the controller and the client software underwent major revisions for the upgraded system.

Siemens made several changes in electronic hardware. Siemens primarily upgraded the high frequency generator from $5 \mathrm{MHz}$ to $40 \mathrm{MHz}$ clock rates. The controller CPU and Linux operating system were upgraded to a $2000 \mathrm{MHz}$ CPU and now runs the 2.6 version of the Debian free GNU variation of Linux. The new controller changed from the ISA bus back plane to a mixed back plane supporting both ISA and PCI modules. This allowed Siemens to use legacy hardware for non-high speed functions and move critical higher speed operations to the faster PCI back plane. The Ethernet interface card was upgraded to support 1 gigabit data transfer rates and automatic sensing of the cabling to determine if connection to the client computer was direct or through a hub. This modification relaxed the requirement for a cross over Ethernet cable. The temperature controllers were modified to improve thermal control on the oven, valve, and inlet. 


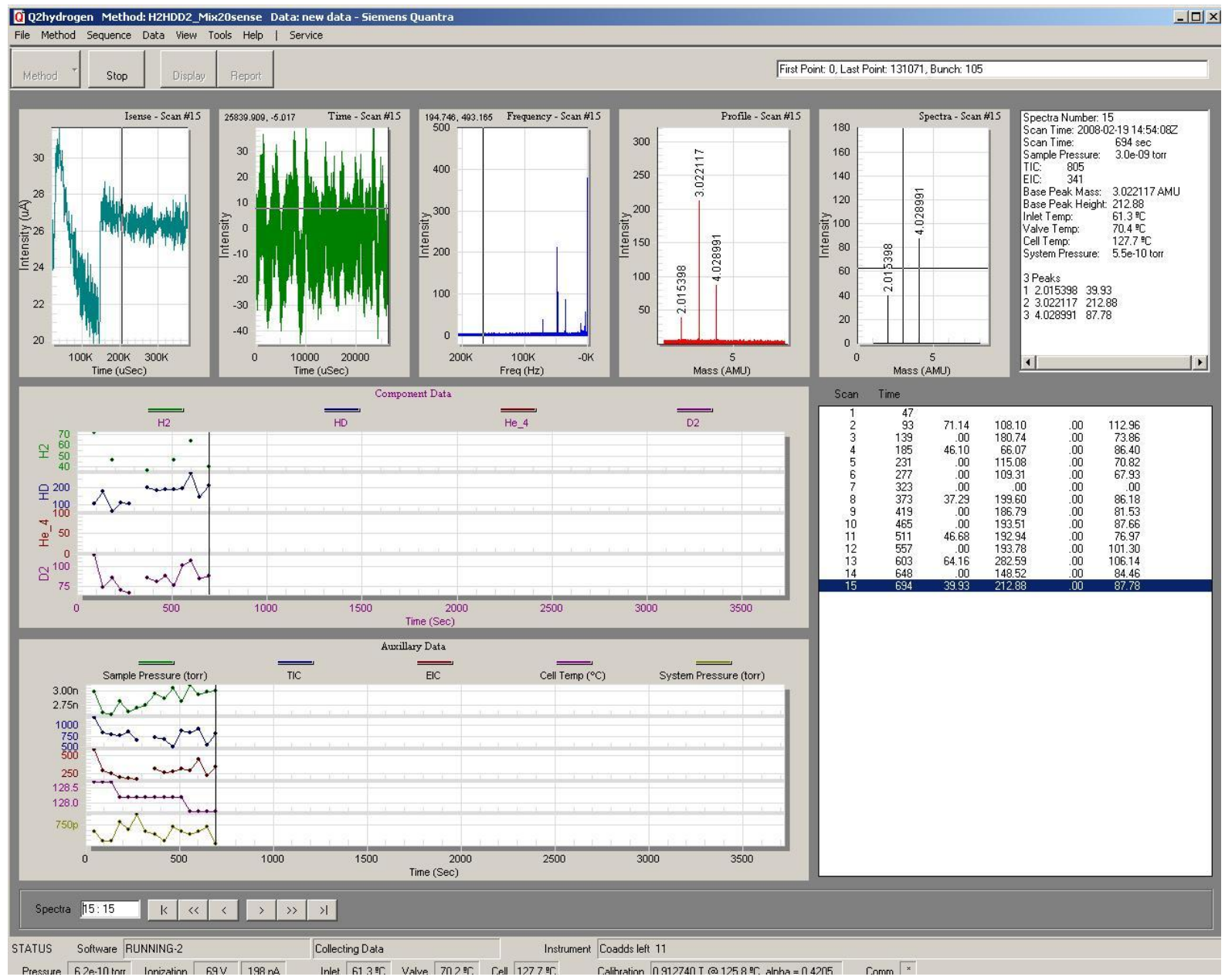

Figure 2 The new client computer interface shows spectra and time trend data on a complex viewing screen. 


\subsection{RESULTS}

The primary hope for the new generation Quantra was to be able to shift the useful working range from mass 6 to 500 down to mass 1 to 500 by increasing the available working frequencies. In the Quantra trap, the sampling frequency (in MHz) times the mass (in atomic mass units (AMU)) should equal a constant that is about 14 MHZ-AMU. As seen in Figure 3, higher mass signals get increasingly closer together at lower frequencies. Resolution is limited by the ability to resolve differences in frequencies.

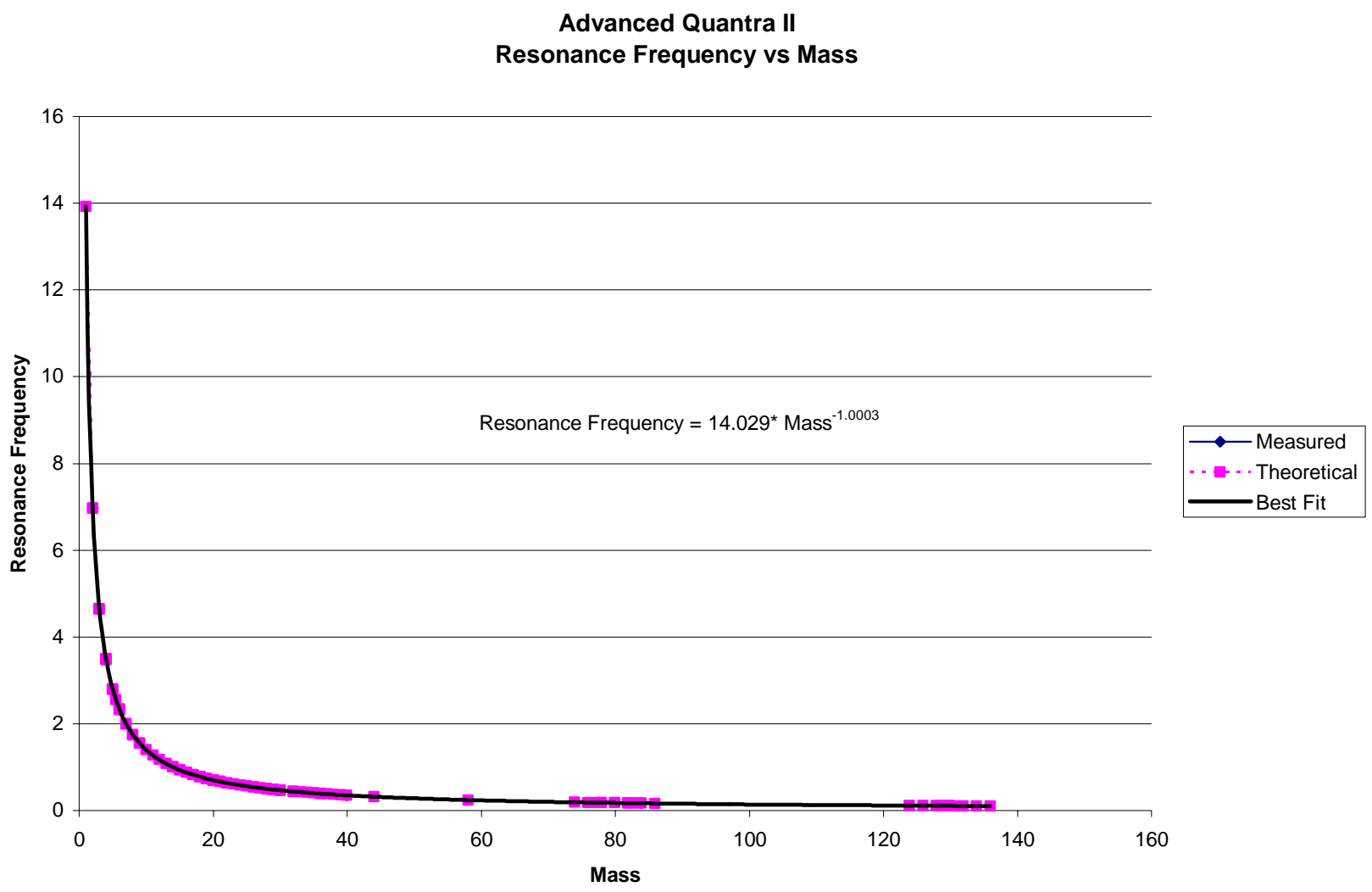

Figure 3 Resonance frequency as a function of mass.

The original Quantra had a clock frequency of $5 \mathrm{MHz}$. Therefore, the minimum mass that could be detected was about 5.6 AMU. The new generation Quantra changed the waveform generator from 5 $\mathrm{MHz}$ to $40 \mathrm{MHz}$. This means that the new minimum detectable mass is approximately 0.7 AMU.

In Siemens's initial evaluation of the new instrument, Dean Davis found that even though the new sampling rate was capable of detecting the signal from the low mass ions, the ion motion in the trap for the light elements was so different that their trap could not accommodate the ions using a single set of applied voltages. In the instrument delivered to SRNL, the best trap potentials for masses were experimentally found as shown in Table 2 for a $70 \mathrm{~V}$ electron beam potential. The scan from 1 to 45 uses compromise conditions between the low and high mass settings and can be used as a check sweep for some inert gases. However, it was found that hydrogen in the presence of non-inert gases such as 
nitrogen and oxygen tends to react rather than give a correct quantitative value. Ejection of reactive ion species from the trap had to be performed to get valid quantitative scans of hydrogen.

Table 2 Optimum Conditions for Mass Ranges

\begin{tabular}{||l|l|l|l|l||}
\hline & $\begin{array}{l}\text { CELL RADIUS } \\
\text { (CM) }\end{array}$ & $\begin{array}{l}\text { IONIZATION TRAP } \\
\text { POTENTIAL (V) }\end{array}$ & $\begin{array}{l}\text { DETECTION TRAP } \\
\text { POTENTIAL (V) } \\
\text { (V) }\end{array}$ & $\begin{array}{l}\text { ELECTRON } \\
\text { BEAM } \\
\text { (NA) }\end{array}$ \\
\hline Mass 1 to 6 & 0.6 & 0.6 & 4.25 & 200 \\
\hline Mass 10 to 500 & 1.5 & 1.5 & 1.4 & 80 \\
\hline Mass 1 to 45 & 1.5 & 0.6 & 2.0 & 200 \\
\hline
\end{tabular}

A possible explanation for the differences in the applied potentials is to consider the diffusion differences between the light and heavy masses. Hydrogen, when injected into the trap, rapidly expands. A lower potential voltage on the trap during the ionization step seems to spread out the ionization beam and allow more hydrogen atoms to be ionized. Because the beam is more spread out, the electron beam density is less and therefore the total beam current had to be increased. On the other hand, heavier gases might have less dispersion. A more focused electron beam can then be used with less beam current to provide the same ionization density. Once the ions are formed, the more highly dispersed light hydrogen atoms require a higher potential to confine them to the trap where as the heavier atoms need weaker applied energy to maintain them in the trap. A discussion of this concept with Davis suggested that the explanation made sense but Siemens believed that the heavier ions were also filling the trap in the time allowed for dispersion. Davis said they were not able to prove the dispersion. A future study could use ion flight models to explain and optimize the voltage settings.

Davis found that the new system could not scan continuously from mass 1 to 500 without further development of the control algorithms. However, he found that it was possible to optimize the scan for low masses, and then change conditions and scan the higher mass range. This may be done automatically using functionality currently in the software. Testing at SRNL indicated that a partial range scan could be done from mass 1 to 45 with some compromise in sensitivity at both the high and low mass range. An attempt to scan up to mass 88 using compromise conditions between the low and high mass settings resulted in highly distorted peak shapes at the high mass end.

Resolution in the ion trap was phenomenal as expected for an FTICR system. At the highest settings, resolution for hydrogen and helium was above 400,000 and 200,000 respectively. Reaching these extremely high resolutions required special attention to be paid to temperature control and optimal operating conditions. The mass peaks are so narrow that even the slightest shift in conditions will result in the peak shifting. As a result, large co-additions at high resolution often resulted with the appearance of several minor satellite side peaks that form at discrete step intervals about the central mass. These side lobes seem to be dependent upon very slight drifts in temperature. Higher resolution requires a longer data acquisition time which leaves the instrument more vulnerable to thermal gradients. The new Quantra greatly improved the temperature control and the stability of masses compared to the first generation units, however further improvement would be helpful especially in those cases where resolution was being pushed above 40,000 for helium. We found that setting the instrument to a resolution below 5,000 resulted in stable peak analysis. The previous instrument stability occurred at resolutions below 1000 . Figure 4 shows an example of the separation of deuterium from helium at mass 4 . 


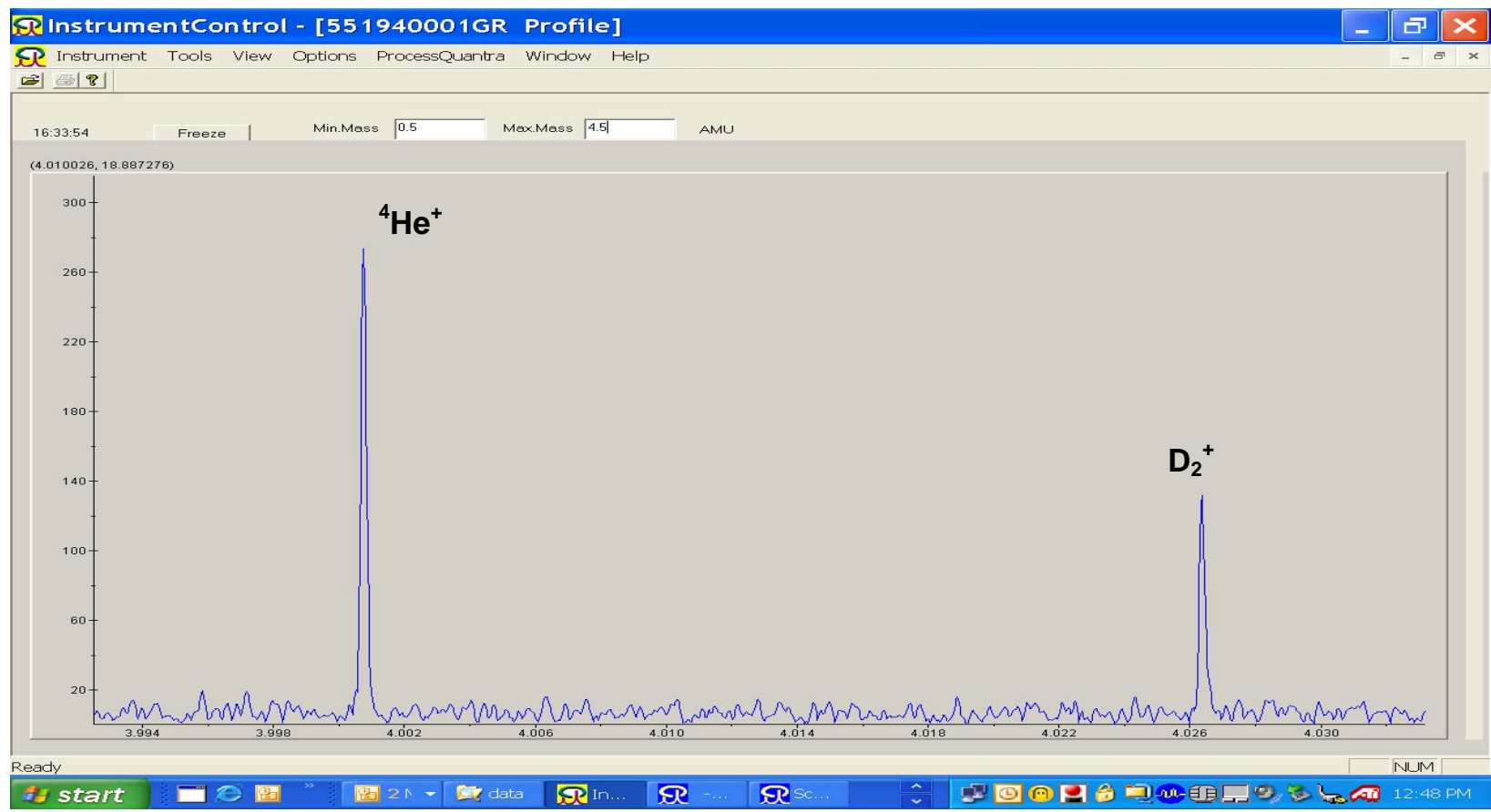

Figure 4 Resolution of ${ }^{4} \mathrm{He}$ from $\mathrm{D}_{2}$ was far superior compared to other mass spectrometers in the SRNL laboratory.

One problem encountered with the new system is the default temperature settings which can be reset by the software program. In the case of a client computer crash, the embedded system shifts the temperature controller set points back to default temperatures that were often different from the current soft settings. When this occurred, stable mass calibrations could not be achieved until the temperature controllers had returned the system back to the desired set point after a client computer reboot. It might be desirable to decouple the temperature controllers from the computer systems using independent controllers. This could be easily done using small digital RTD thermostats like the Watlow controllers that are commonly used in SRNL labs. Siemens is aware of this issue and is currently working on software control options that improve thermal control of these default temperature settings.

Trap loading conditions were critical to valid measurements. Overloading the trap will result in the formation of $\mathrm{MH}^{+}$ions. Figure 5 shows the mass spectrum obtained for an equilibrium mixture of hydrogen and deuterium with optimum cell loading. A 50:50 blend of hydrogen and deuterium was passed through a palladium diffuser to bring the two gases into atomic equilibrium. Theoretically, the resulting mix should be $26.3 \% \mathrm{H}_{2}, 47.4 \% \mathrm{HD}$, and $26.3 \% \mathrm{D}_{2}$. The same gas mixture was analyzed with the JEOL GCMATE to confirm the gas concentrations.

As seen in figure 5, the hydrogen response is slightly less than the deuterium. This could be could be due to differences in either ionization or that hydrogen is not held in the trap as well as deuterium. The difference is small enough though that the system can be used to check for equilibrium with only a small data correction. The spectrum shows that a small amount of $\mathrm{HD}^{++}$could be detected at mass 1.5. If the instrument was set to a lower resolution, the hydrogen signal increased indicating that some of the response was due to ion interactions that were trap time dependent. 


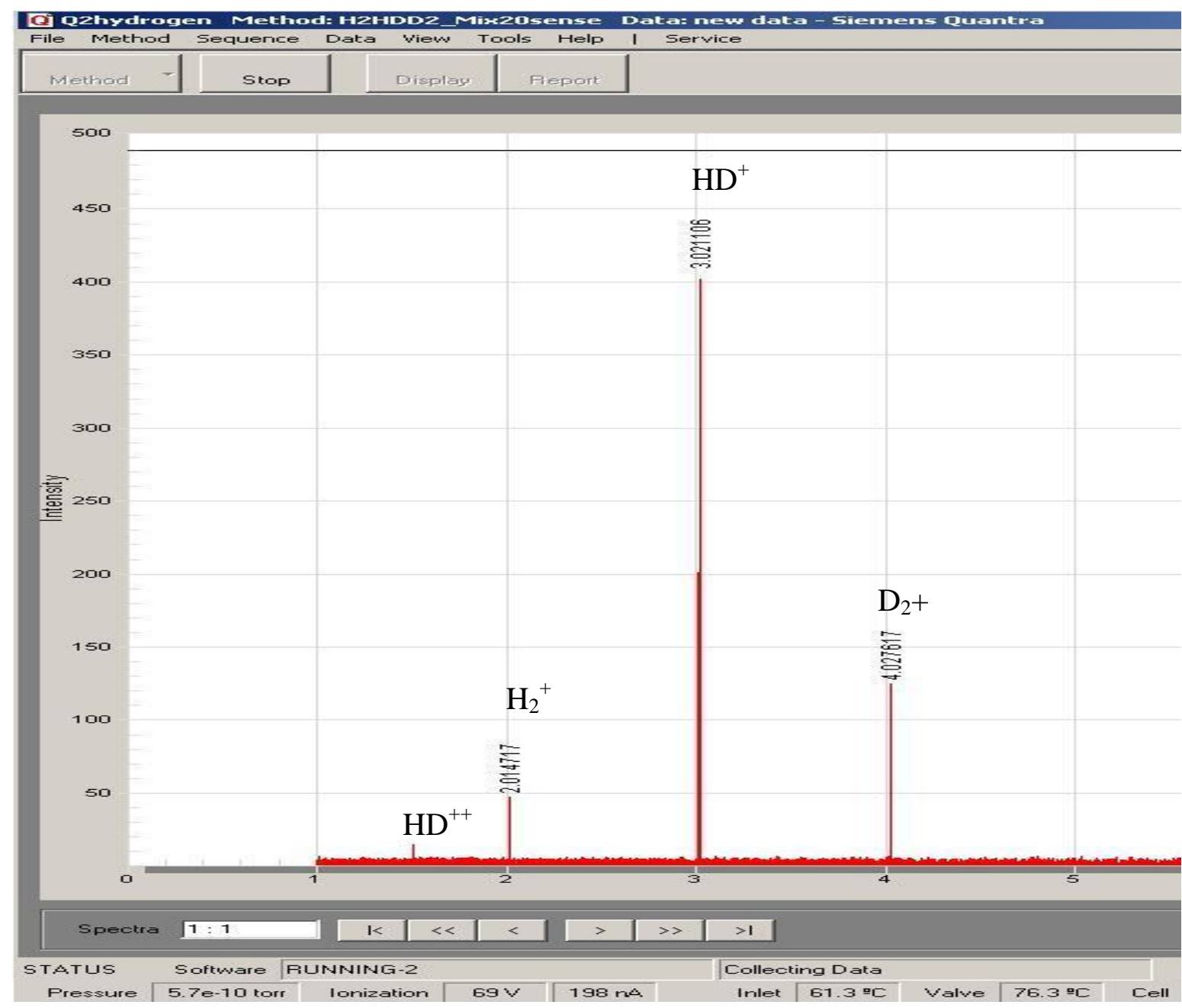

Figure 5 Hydrogen response was less than deuterium in the equilibrium blend of equal portions of the gases. This could be due to differences in ionization or that the hydrogen was not being confined in the trap as well as the deuterium.

Slightly overloading the trap resulted in significant changes to the spectra due to the chemical ionization effect that is commonly observed in many ion traps. Figure 6 shows the same mixture as in Figure 5 with the trap loading increased by an additional 50\%. The image now shows masses at 5 and 6 AMU, which are attributed to formation of $\mathrm{HDD}^{+}, \mathrm{D}_{2} \mathrm{H}^{+}$and $\mathrm{D}_{2} \mathrm{D}^{+}$. The background in this image shows the level of noise when only 2 scans are averaged. Background signal tended to decrease by the square root of the number of scans. Allowing the system to co-add spectra above 128 scans reduced the background by an order of magnitude. A single scan took about 2 seconds so low noise spectra took only about 5 minutes. The detection limit without ejection of major ions was limited to about 1000 ppm. Ejection of major ions would lower the detection limit to around $100 \mathrm{ppm}$. Carbon dioxide levels in air (0.0314\%) could be readily detected using ion ejection. 


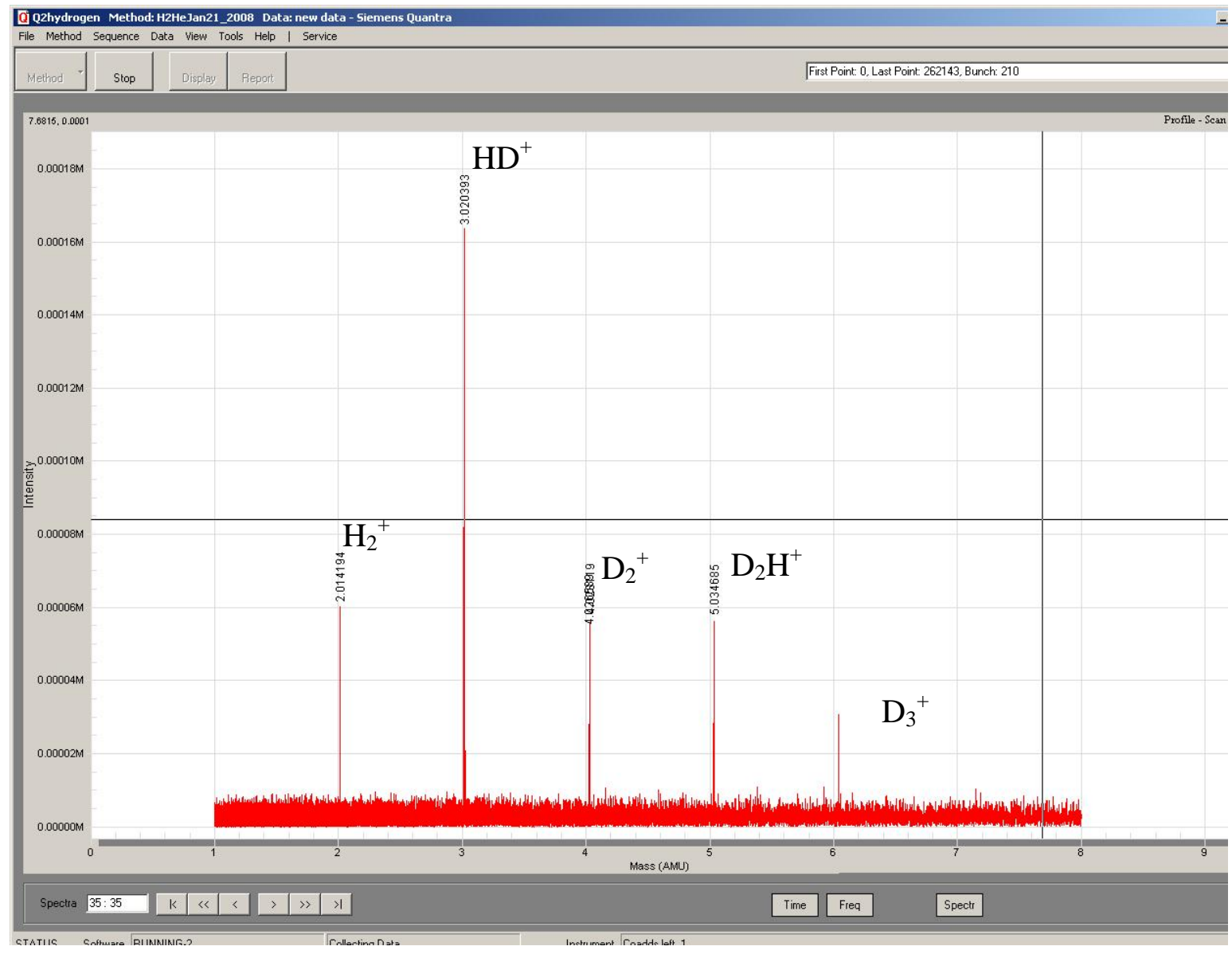

Figure 6 The molecular ion tended to protonate $\left[\mathrm{MH}^{+}\right]$or deuterate $\left[\mathrm{MD}^{+}\right]$when the trap was overloaded or a reactive species was present. Note the presence of ions at mass 5 and 6 in the mix of hydrogen and deuterium. Background noise level in this scan could easily be reduced using multiple co-added scans.

Resolution of the spectrometer was a function of the mass and controlled by the attainable resolution in frequency steps. A resolution of 10,000 at mass 4 corresponds to only about a resolution of 1000 at mass 84 as seen in figure 7 for a scan of the krypton isotopes. Using a high number of co-adds (128), the small $0.35 \%$ mass 78 isotope was observed in the presence of the other krypton isotopes. The detection limit was found to be about $0.1 \%$. Lowering the detection limits to around $30 \mathrm{ppm}$ could be achieved by careful ejection of the major non-essential masses and increasing the electron beam current. Lowering the detection limits should be possible by making use of pre-concentration methods.

Figure 8 shows a mixture of $\mathrm{H}_{2}, \mathrm{HD},{ }^{4} \mathrm{He}$, and $\mathrm{D}_{2}$. The graphic display of the computer limited the visible resolution. To enhance the view of extremely narrow or closely spaced peaks, the zoom function of the software needed to be used. Figure 7 shows the expanded view of the krypton isotopes. 


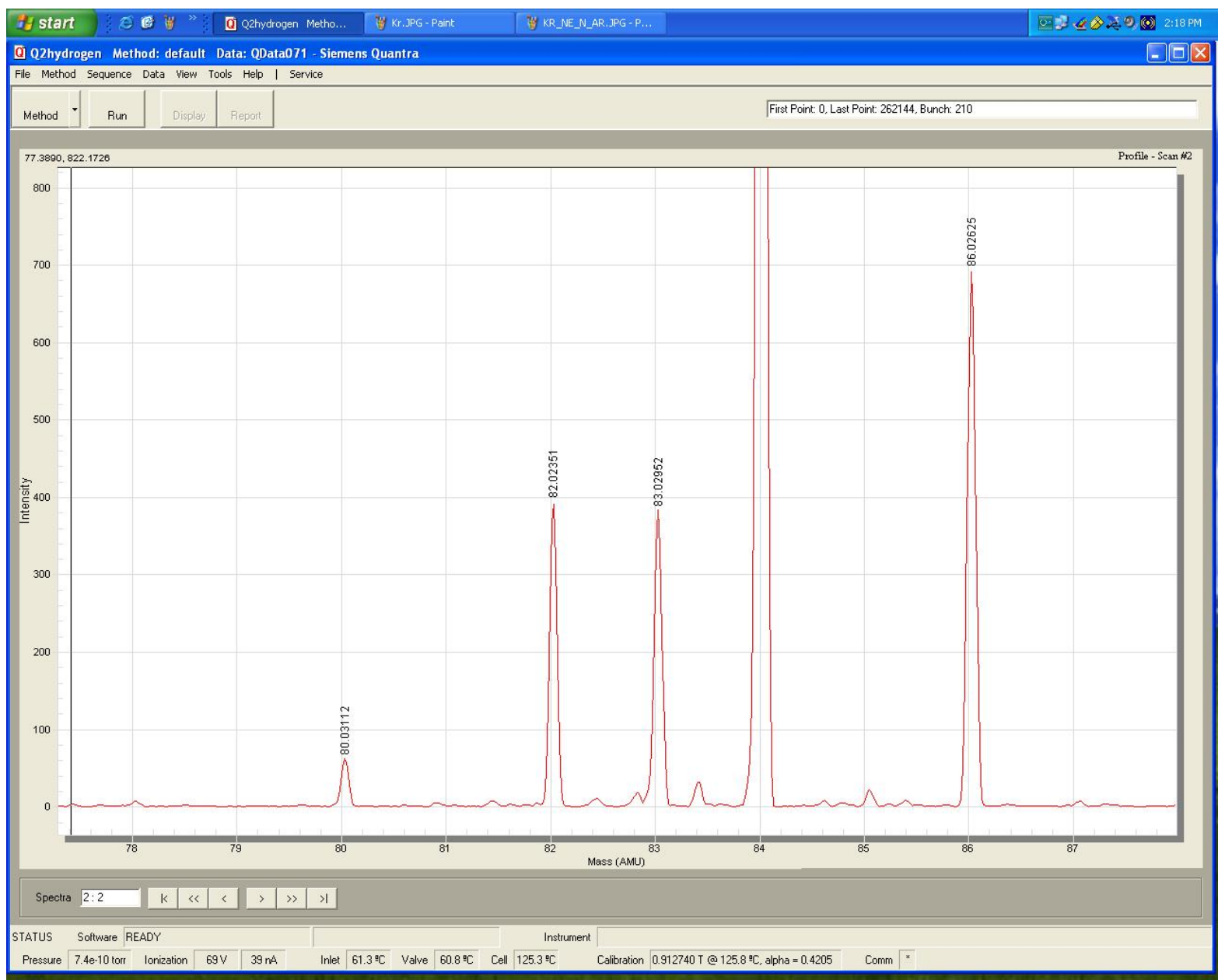

Figure 7 Minor isotopes of krypton and their relative abundance are shown; $\mathbf{m} 78 / 0.35 \%, \mathbf{m 8 0} / 2.28 \%, \mathrm{m82}$ $11.6 \%, \mathrm{m83} / 11.5 \%, \mathrm{m84} / 57.0 \%$ [off scale], m86/17.4\%. This image had 128 co-adds to decrease noise. Masses calibration is slightly shifted. 


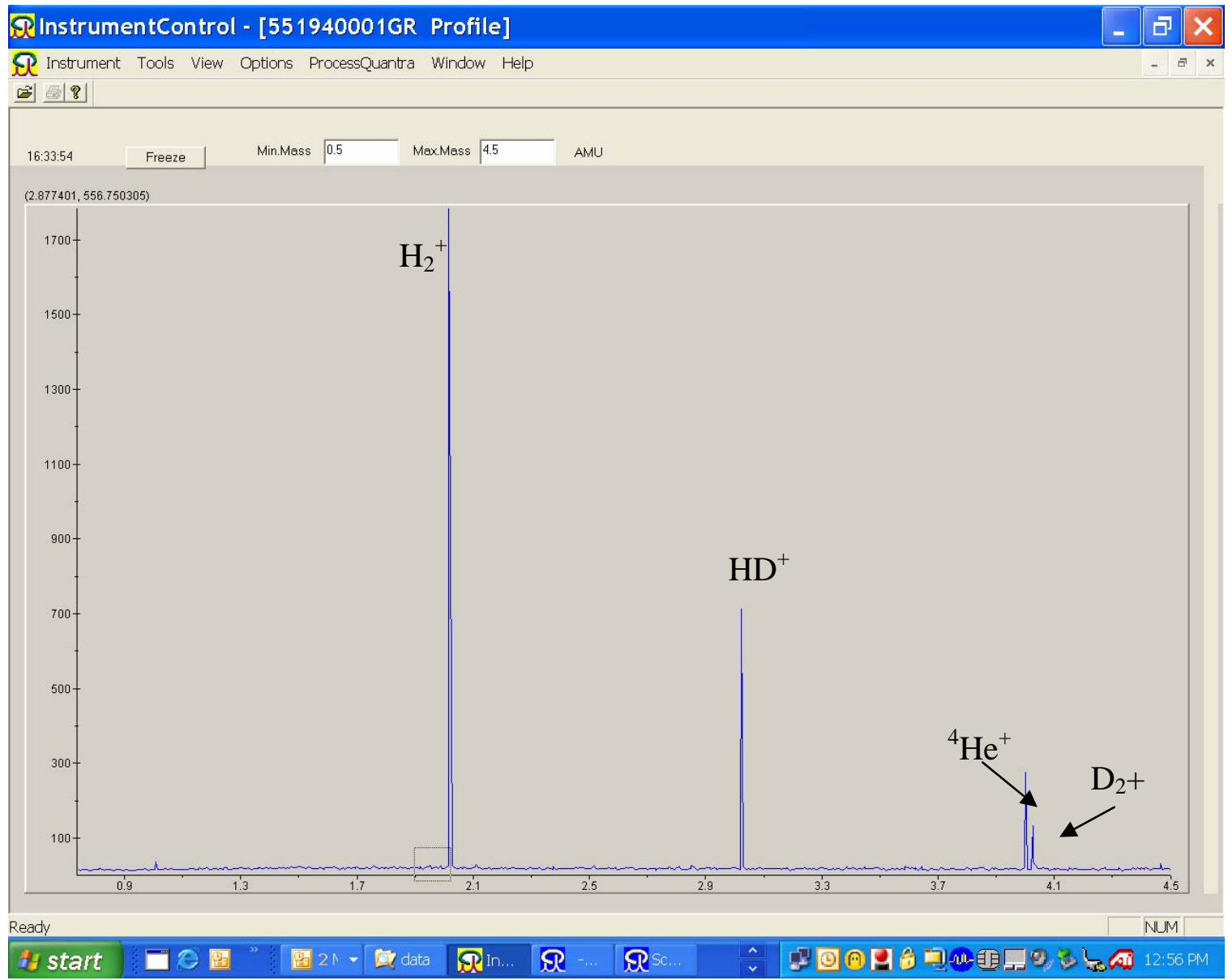

Figure 8 Spectrum shows a mixture of $\mathrm{H}_{2}, \mathrm{HD},{ }^{4} \mathrm{He}$ and $\mathrm{D}_{2}$.

The new software improved the ability to monitor ions as a function of time. Up to 4 ions could be tracked in real time on the computer screen with the beta software versions used in our evaluation. Figure 9 shows a screen capture of the dilution of neon gas over time as the sample was displaced using a small in leakage of air. The neon-20 mass decreases and the nitrogen-28 mass increases. This data was collected at a moderate 10,000 resolution setting. At this resolution, the beta version of software occasionally lost its lock on the mass or data set and a spike occurred in the data. The exact reason for these spikes is not known. Without the noise spikes, the data indicates about a $5 \%$ deviation for repeated signals. It might be possible to improve the precision by optimizing the piezo-electric injection parameters. Otherwise a lock to an internal reference species would be needed to improve measurements for quantization. 


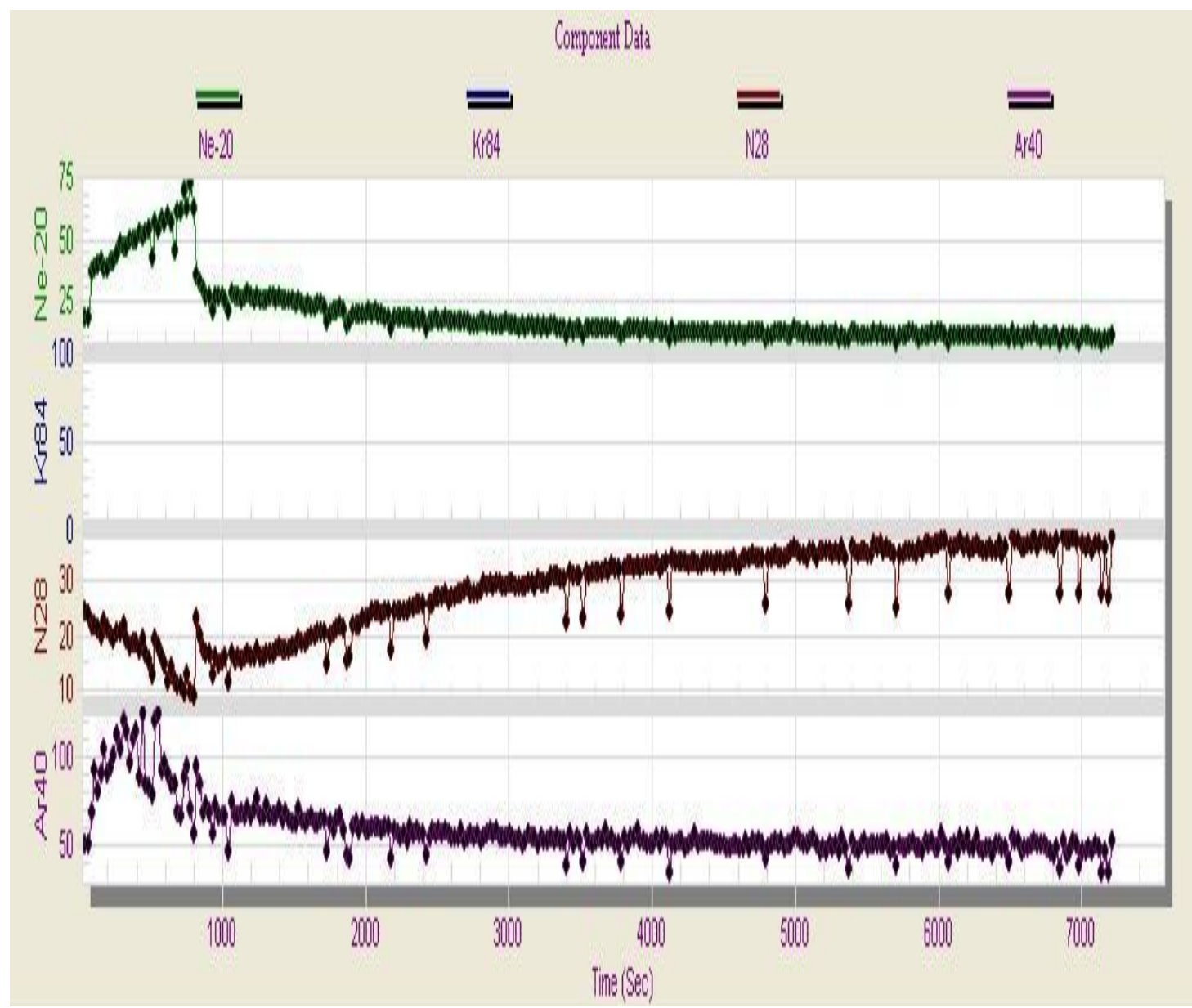

Figure 9 A close look at trend data taken at high resolution shows that the spectrometer would occasionally lose its mass lock and create a single point spike in the data set.

Software stability remains an issue with the Siemens software and appears to be related to the use of an Ethernet interface as the data transfer option. Data could be collected using restricted operating conditions for long 16 hour data collection runs at moderate resolution. Testing showed that when logged on to the site domain network and then running the Siemens instrument that the instrument rarely could run for more than a few data cycles before a system crash occurred. Best performance occurred when logged on as the local machine administrator and restricting site internet hidden processes such as automatic antivirus and other software updating services. System crashes occurred even when the site network interface card was disabled. The problem appears to be associated with the number of background svchost.exe processes that are attempting to run on the network system and we found that many of those processes are very difficult to shutdown in our networked systems. System crashes were much more prevalent at high resolution when larger, time critical data transfers occur. An alternative to ethernet data links might be required for reliable performance. Siemens is aware of the problem and is investigating options for improving the performance of the data handling system. The new software greatly improved the ability of the system to recover from a data communication crash once it occurred. A complete reboot of both systems was rarely required and the software appeared to reset to standby conditions after a three minute timeout. Unfortunately, as mentioned above the reset also disturbed the instrument temperature control which was critical to high resolution stable measurements. A slight change in temperature results in shifted and unstable peak measurements. Most of these problems will likely be solved in future software and firmware revisions. Only beta release versions of the control software were available during this evaluation. 


\subsection{CONCLUSIONS}

The new Siemens Advanced Quantra demonstrated phenomenal resolution in the low mass range. Resolution greater than 400,000 was achieved for mass 2 . The new spectrometer had a useful working mass range to 500 Daltons. However, experiments found that a continuous single scan from low mass to high was not possible. Two useful working ranges were established covering masses 1 to 6 and masses 12 to 500 for our studies. A compromise performance condition enabled masses 1 to 45 to be surveyed. The instrument was found to have a dynamic range of about three orders of magnitude and quantitative analysis is expected to be limited to around 5 percent without using complex fitting algorithms.

Analysis of low concentration ions, at the ppm level, required a separate analysis using ion ejection techniques. Chemical ionization due to the formation of the $\mathrm{MH}^{+}$ion or $\mathrm{MD}^{+}$increased the complexity of the spectra compared to magnetic sector mass spectra and formation of the protonated or deuterated complex was a dynamic function of the trap ion concentration. This made quantitative measurement more of a challenge.

However, the resolution of the instrument was far superior to any other mass spectrometry technique that has been applied to the analysis of the hydrogen isotopes. The piezo-electric picoliter injection device offers a new way of submitting small quantities of atmospheric pressure sample gas for analysis. The new software had many improvements over the previous version but significant flaws in the beta codes remain that make the prototype units less than ideal. The instrument is a promising new technology that experience will likely improve. Unfortunately, Siemens has concluded that the technology will not be a commercial success and has decided to stop producing this product. 
WSRC-STI-2008-00161

Rev. 0

This page intentionally left blank

Page 16 of 22 


\subsection{RECOMMENDATIONS/PATH FORWARD}

Siemens has notified NNSA that they would not support the Quantra unless they found a market that would support significant annual sales. Because Siemens does not intend to support the instrument as a commercial offering, SRNL recommends limiting development work with the instrument.

The Quantra will be most useful as a complement to, not a replacement for, the existing mass spectrometers. Because of the instrument's superior resolution, it would be especially beneficial for measuring analytes that are difficult to resolve with the currently used mass spectrometers. For example, it could be used to monitor $\mathrm{CO}$ and $\mathrm{N}_{2}$ concentrations directly, which is a common support request and a very difficult conventional mass spectrometry measurement. The instrument can be used to measure mass 16-20 isomers such as oxygen, ammonia, water, or methane. For measurement of the hydrogen isotopes as well as other analytes in a hydrogen isotope matrix, method development and parameter optimization will be required to understand and minimize the chemical ionization that occurs in the ion trap. Testing with tritium will be needed to evaluate and understand its interactions in the ion trap. Finally, the Siemens Quantra may be used for training and demonstration of high resolution residual gas mass measurements with ion traps.

Development of quantitative algorithms and more robust crash resistant operating codes would make the Quantra system suitable for process support applications. Siemens will likely have at least one revision of their software to evaluate. An alternative for Quantra instrument control and data analysis would be to apply the Predator System [ref 13] developed at the National Magnetic Laboratory at Florida State University.

SRNL will monitor external developments and literature with Siemens and other manufacturers of FTICR systems. While this report was being reviewed, Ivey and Hemminger [ref 14] published a paper characterizing the issues associated with the detection of hydrogen by FTICR which are in agreement with the SRNL observations discussed in this report. The authors observed and discussed two mechanisms that affect the hydrogen signal: 1) z-ejection and 2) proton and chargetransfer reactions in the trap. They also demonstrated that parameters could be optimized to minimize these effects for quantitative measurement of hydrogen. 
WSRC-STI-2008-00161

Rev. 0

This page intentionally left blank

Page 18 of 22 


\subsection{REFERENCES}

1. Phillipe Chastagner, Advanced Mass Spectrometers for Hydrogen Isotope Analysis, DP-1674, June 1984.

2. W.A. Brand, T.B. Coplen, “An Interlaboratory study to test instrument performance of hydrogen dual-Inlet isotope-ratio mass spectrometers, Fresenius J. Anal. Chem. (2001) v370, p358-362.

3. Alex L. Sessions, Thomas W. Burgoyne, and John M. Hayes, "Correction of H3+ Contributions in Hydrogen Isotope Ratio Monitoring Mass Spectrometry”, Anal. Chem. (2001), V73, p 192-100

4. David Demange, Mauel Grivet, Hubert Pialot, and Alain Chambaudet, “Indirect Tritium Determination by an Original 3He Ingrowth Method Ising a Standard Helium Leak Detector Mass Spectrometer”, Anal. Chem. (2002), V74, p3183-3189.

5. Joe Cordaro and John McIntosh, "Electronics Upgrade of High Resolution Mass Spectrometers”, WSRC-STI-2007-00658. Presented at the $54^{\text {th }}$ International Instrument Symposium, ISA 2008 [www.isa.org].

6. L.L. Tovo, R.J. Lascola, W.A. Spencer, C.S. McWhorter, and K.E. Zeigler, “ Evaluation of Technologies to Complement/Replace Mass Spectrometers in the Tritium Facilities(U)”, WSRCTR-2005-00409, Rev 0, p 1-61, August 30, 2005.

7. Alan G. Marshall, Christopher L. Hendrickson, and George S. Jackson, “ Fourier Transform Ion Cyclotron Resonance Mass Spectrometry: A Primer”, Mass Spectrometry Reviews (1998), V 17, p $1-13$

8. Bruce Asamoto, editor, FT-ICP/MS: Analytical Applications of Fourier Transform Ion Cyclotron Resonance Mass Spectrometry, VCH Publishers, New York, 1991, (ISBN 0895737671, ISBN 3527279199). library \# QD96.I54F78

9. M.V. Buchanan, Ed., Fourier Transform Mass Spectrometry: Evolution, Innovation and Applications, ACS Symposium Series, 359: American Chemical Society: Washington, D.C. 1987.

10. L.L. Tovo FTICR Specification -J-SPP-A-00029, September 27, 2005, p 1-19.

11. Q. G. Grindstaff, "Future Improvements in Gas Sampling and Analysis”, Presented at the NNSA Future Technologies Conference 2004.

12. Q. G. Grindstaff, Y-12, personal communication, 2005.

13. Greg T. Blakney, Christopher L. Hendrickson, John P. Quinn, and Alan G. Marshall, "New Hardware for Ultrahigh Resolution SWIFT Ion Isolation in an FT-ICR Mass Spectrometer", TP018, 56 ${ }^{\text {th }}$ American Society for Mass Spectrometry Annual Conference on Mass Spectrometry, Denver, CO, June 1-5, 2008.

14. Michelle M. Ivey and John C. Hemminger, "Simultaneous Detection of $\mathrm{H}_{2}{ }^{+}$with Larger Ions Using Fourier Transform Mass Spectrometry”, Anal. Chem. (2008), V80, p 5044-5050. 
WSRC-STI-2008-00161

Rev. 0

This page intentionally left blank

Page 20 of 22 


\subsection{ACKNOWLEDGEMENTS}

We wish to thank Dean Davis of Siemens Applied Automation Division, Bartlesville, OK, for patiently explaining the system, software, limitations, and discussing possible uses for the instrument. His prompt response and support are gratefully acknowledged.

Henry (Tommy) Sessions, Sharon Redd and others in the SRNL Hydrogen Processing Group provided significant help by providing supply gases, as well as calibrated $\mathrm{H}_{2}, \mathrm{HD}$, and $\mathrm{D}_{2}$ equilibrium mixtures. They also shared the occasional needed gas fitting.

Dr. John Gill, LANL, and Dr. Robert E. Ellefson, Consultant/ Mound Technical Solutions, helped with the field assessment at Bartlesville, OK, and Dr. Quirinus Grindstaff, Y-12, helped with technical review and several discussions about instrument specifications and performance criteria. 


\section{Distribution:}

M. J. Barnes, Analytical Development

L. M. Chandler, Analytical Development

P. F. Cloessner, Defense Programs Technology

K. L. Sessions, Defense Programs

W. A. Spencer, Analytical Development

L. L. Tovo, Analytical Development

S. B. Wyrick, Defense Programs Technology

R. L. Rabun, Defense Programs Engineering

C. B. Mauldin, Tritium Labs

R. C. Hooper, Tritium Labs

J. L. Clark, Defense Programs

E. L. Hart, Tritium Labs

P. Cable-Dunlap, Non-Proliferation Tech

J. E. Halverson, Non-Proliferation Tech

C. R. Shick, Non-Proliferation Tech 